\title{
The Dynamic Origin of Color Tuning in Proteins Revealed by a Carotenoid Pigment
}

\author{
Daniele Loco, ${ }^{\dagger}$ Francesco Buda, ${ }^{*, \ddagger}$ Johan Lugtenburg, ${ }^{\ddagger}$ and Benedetta \\ Mennucci*, ${ }^{*}$ \\ $\dagger$ †ipartimento di Chimica e Chimica Industriale, University of Pisa, via G. Moruzzi 13, \\ 56124, Pisa, Italy \\ $\ddagger$ Leiden Institute of Chemistry, Leiden University, Einsteinweg 55, 2300 RA Leiden, The \\ Netherlands \\ E-mail: f.buda@chem.leidenuniv.nl; benedetta.mennucci@unipi.it
}




\begin{abstract}
Understanding the microscopic origin of the color tuning in pigment-protein complexes is a challenging yet fundamental issue in photoactive biological systems. Here, we propose a possible interpretation by using a state-of-the-art multiscale strategy based on the integration of quantum chemistry and polarizable atomistic embeddings into a dynamic description. By means of such a strategy we are able to resolve the long-standing dispute over the coloration mechanism in the crustacyanin protein. It is shown that the combination of the dynamical flexibility of the carotenoid pigments (astaxanthin) with the responsive protein environment is essential to obtain quantitative predictions of the spectral tuning. The strong linear correlation between the excitation energies and the bond length alternation in the long-chain carotenoids modulated by the dynamical protein environment is a novel finding explaining the high color tunability in crustacyanin.
\end{abstract}

Color tuning of chromophores by the embedding protein is an extremely effective strategy that nature has developed and optimized to activate different biological functions. ${ }^{1}$ Many examples are known from simple microorganisms to complex systems and various mechanisms have been suggested both from experimental and theoretical investigations. ${ }^{2-14}$ These mechanisms are mostly based on the electrostatic interactions of protein residues surrounding the chromophore that, either through net charges or large polarity effects, can differentially stabilize ground and excited states. The other mechanism which is often suggested is the conformational change of the chromophore exerted by the confinement in the protein binding pocket. However, in most cases, electrostatics and conformational effects can only qualitatively explain the observed color change. ${ }^{11,15,16}$ It is therefore more likely that multiple effects are simultaneously active making the elucidation of the mechanism of tuning still an unsolved problem. Here we show that an exhaustive explanation is indeed achievable through advanced computational approaches.

The specific case here selected is one of the largest and most spectacular color changes induced by a protein matrix. When heating the lobster (Homarus Gammarus), the dark blue color of its carapace is changed into bright red. Lobster carapaces contain a ketocarotenoid pigment called astaxanthin (AXT), which, when embedded in the $\alpha$-crustacyanin protein, gives a $\pi-\pi^{*}$ absorption maximum at $\lambda_{\max } \sim 630 \mathrm{~nm}$. Upon protein denaturation, the released AXT appears as a red pigment, with a maximum absorption between 475 and $500 \mathrm{~nm}$, depending on the solvent. Although it is clear that the color change is due to the formation of the carotenoid-protein complex, the exact mechanism inducing this large spectral shift has not been established. The elucidation of the crystal structure of the dimeric subunit of the protein $(\beta \text {-crustacyanin })^{17}$ has revealed the 6-s-trans conformation of AXT in the binding pocket, contrary to the 6-s-cis conformation observed in solution. The X-ray structure of $\beta$ crustacyanin has also shown the close proximity of the two AXT molecules in the dimeric subunit (Figure 1) with a center-to-center intermolecular distance of about $7 \AA .{ }^{17}$ Moreover, the AXT carotenoids appear to be bent within the binding pocket and their carbonyl groups form hydrogen bonds with a nearby histidine and a water molecule, respectively.

Such X-ray structural information has further stimulated several theoretical and experimental investigations. ${ }^{10,11,15,16,18-22}$ The origin of the large bathochromic shift of the order of $0.5 \mathrm{eV}$ has been related to (i) planarization of the chromophore in the protein binding pocket inducing an extension of the conjugated system; (ii) polarization effects due to either charged residues in the vicinity of the chromophore or due to long-range polarization effects; (iii) excitonic coupling due to the proximity of the two bound chromophore (as also suggested by CD spectra ${ }^{15,23}$ ), or a combination of the above mechanisms. The hypothesis of a strong excitonic splitting, originally proposed on the basis of a simple dipole-dipole model approximation, ${ }^{15}$ has been more recently discarded based 
on more accurate theoretical calculations ${ }^{16,21}$ as well as 2D electronic spectroscopy investigations, which give an exciton coupling of 250 $\mathrm{cm}^{-1}(0.03 \mathrm{eV}) .^{22}$

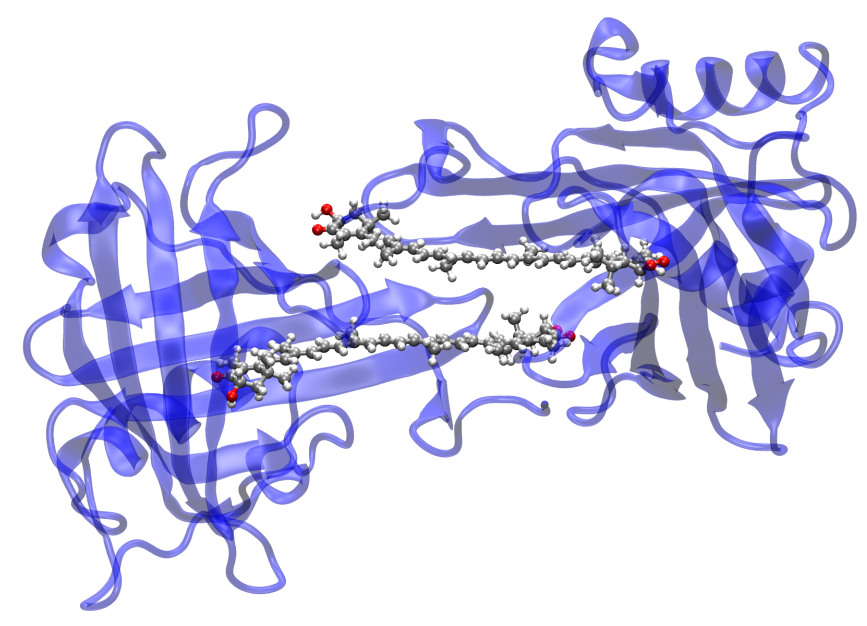

Figure 1: $\beta$-crustacyanin protein scaffold with the two AXT carotenoids inside it, in the configuration represented in the cristallographic structure. ${ }^{17}$

Another long-debated idea is the presence of a net charge on the AXT end rings or a nearby charged residue inducing a strong charge polarization of the conjugated chain, thus explaining the large spectral shift compared to the chromophore in nonpolar solvent. ${ }^{21}$ Recently, a work combining experimental data and quantum-chemical calculations on a model compound has proposed the enolization of AXT within the protein environment to explain the large bathochromic shift. ${ }^{10}$ However, it is difficult to reconcile this suggestion with the solid state ${ }^{13} \mathrm{C}$ NMR data on $\alpha$-crustacyanin that have shown rather small chemical shifts differences compared to ${ }^{13} \mathrm{C}$ NMR data in solution. ${ }^{15,16}$ These data quite convincingly indicate no evidence of enolization that would result in much larger chemical shifts.

In a recent computational study, GamizHernandez et al. employed an hybrid QM/MM approach using correlated ab initio calculations for the excitation energies and they showed the importance of properly including the specificity of the different protein residues surrounding the pigment. ${ }^{11}$ Their predicted color shift of about $0.3 \mathrm{eV}$, however, accounts for about $60 \%$ of the observed experimental shift from hexane to $\beta$ crustacyanin. The question is what are the missing elements in the theoretical model for a quantitative prediction?

In this work, we provide the answer by comparing a hierarchy of models of increasing completeness which combine a quantum chemical characterization of the structure, the dynamics and the optical response of the chromophore(s) with a classical description of the environment. This comparative study not only allows us to resolve the long-lasting dispute over coloration mechanism in crustacyanin but it also clarifies the limits of the commonly used computational strategies to study the effects of the protein on the response of the embedded chromophore to light.

It is well known that the quantum chemical description of the electronic excitations in carotenoids is extremely challenging due to the specific character of the different $\pi-\pi^{*}$ excited states. Indeed, from theoretical studies comparing single- and multi- reference approaches, ${ }^{24-27}$ it comes out that contributions from multiple excitations are crucial for the description of the lowest (dark) excited state, whereas the (bright) second state is dominated by single excitations. ${ }^{28}$ For that excitation, Time Dependent Density Functional Theory (TDDFT) has shown to be a valid approach especially when used in combination with optimized long-range corrected hybrid density functionals. This is exactly the level of QM description that will be used here to describe the bright excitation of AXT in terms of the $\omega$ B97x functional. $^{29}$ The validity of the selected functional has been further confirmed by comparing the results obtained for AXT to those of another carotenoid, violoerythrin (VIO), which has the same length of AXT but with isopentenone rings instead of ionone rings. VIO shows already a blue color in hexane solution $\left(\lambda_{\max }=530\right.$ $\mathrm{nm}$ for VIO compared to $\lambda_{\max }=472 \mathrm{~nm}$ for AXT).${ }^{30,31}$ In Section S2 of the Supporting Info (SI) we provide validation tests on the $\omega \mathrm{B} 97 \mathrm{x}$ functional and show that it is able to quantitatively predict the experimental spectral shift for AXT and VIO carotenoids in solution. In the following, all the computed excitation energy shifts will refer to the unbound AXT in cis- conformation in cyclohexane represented through 
the Polarizable Continuum Model (PCM) ${ }^{32}$ In fact, while AXT in the protein crystal is in the trans form, in solution the cis conformation is known to be dominant (see also Table S1 in SI). ${ }^{11,19}$

As a first model for the pigment-protein complex, we assume that the effect of the protein can be mimicked with a dielectric medium that we represent through PCM. To account for the water molecules that can penetrate into the protein and contribute to the polarity of the local environment of each AXT, we have used a dielectric constant corresponding to a solvent of medium-high polarity, namely $\epsilon=20$, in combination with an optical dielectric constant equal to 2.0 as commonly used for proteins. Using such an effective dielectric model, we have optimized the geometry of the single 6-strans AXT in PCM at B3LYP/6-31G(d) level, and computed the excitation energy at the TD $\omega$ B97x/6-31+G(d) level. To properly account for the differential stabilization of ground and excited state we have also introduced a state-specific correction to the TDDFT excitation energies. ${ }^{33}$

The value of $0.17 \mathrm{eV}$ for the bathochromic shift obtained at the QM/PCM level (see Table 1) when moving from hexane to $\beta$ crustacyanin recovers only a portion of the experimental value $(0.51 \mathrm{eV}) .{ }^{17,20}$ This shift is mainly associated to the planarization of the chromophore in the s-trans conformation. What is clearly missing in this "continuum" picture are both the specific interactions between the AXTs and the protein residues and the possible inhomogeneities in the electrostatic and polarization effects of the protein matrix. A possible step forward is thus the inclusion of an atomistic MM method based on the available crystal structure of the pigment-protein complex. Because the resolution of this structure for the chromophore is not enough to be safely used in the QM calculations, we relaxed the geometry of the two AXTs within the protein kept frozen in its crystal structure. The geometry optimization was performed at B3LYP/6$31 \mathrm{G}(\mathrm{d}) / \mathrm{AMBER}$ level using an electrostatic embedding. The resulting geometries were finally used to calculate the excitation energies of the chromophores still within the same electrostatic embedding for the protein. The resulting excitation energy shifts, reported in Table 1 , show that the two AXTs "feel" a quite different local environment: for one (AXT A), the shift is more than double than for the other (AXT $\mathrm{B})$ and even larger than the experimental one. A possible improvement to this description is to combine the same relaxed AXTs structures with a polarizable embedding for the protein. Here the polarizable AMOEBA force field ${ }^{34}$ has been used in its recent implementation within a TDDFT framework. ${ }^{35}$ In such an implementation a state-specific correction of the TDDFT excitation energies can also be included using a formalism similar to what done at PCM level. Through this correction, it is possible to recover the effects due to the relaxation of the chromophore electronic density upon excitation and combine it with those due the response of the polarizable embedding to the excitation (see eq. S7 of the SI). ${ }^{36}$

The resulting shift increases of $\sim 0.1 \mathrm{eV}$ for both AXTs if compared to the non polarizable QM/MM results. As the AXT geometries and the protein configuration have not changed, this additional shift is only due to the combined effect of (i) the state-specific relaxation of the protein polarization to both ground and excited states and (ii) its response to the electronic transition. ${ }^{36}$ In the present case, the former effect is much smaller than the second one, as the $\pi-\pi^{*}$ excitation does not correspond to a significant reorganization of the electronic charge.

To investigate the possible sources of the different shift shown by the two AXTs in the crystal structure, we have dissected the contribution of the various residues surrounding the pigments. Two aspects have been considered: how much each residue contributes to stabilize the ground state (through electrostatics and polarization) and how much it responds to the excitation through polarization (see Section S1 of the SI for more details). The results are reported in Fig.2. As it can be seen, for both AXTs the residues which respond the most to the excitation are not homogeneously distributed around the chromophore but they concentrate more on 
Table 1: Comparison between calculated and experimental excitation energy shifts for different QM/classical models. The shift is obtained with respect to the cis- form in n-hexane (2.56 eV at TD $\omega$ B97x/PCM level and $2.63 \mathrm{eV}$ in the experiments). The experimental value is from Ref. 17,20. All values are in $\mathrm{eV}$.

\begin{tabular}{lc}
\hline Model & Shift \\
\hline PCM & 0.17 \\
MM & $0.59^{a} \& 0.19^{b}$ \\
AMOEBA & $0.72^{a} \& 0.29^{b}$ \\
$<$ AMOEBA $>_{M D}$ & 0.50 \\
\hline Experiment & 0.51 \\
\hline${ }^{a}$ AXT A; ${ }^{b}$ AXT B
\end{tabular}

one side, namely the side with the H-bonded water molecule. However, the relative contributions are different in the two AXTs: in particular, AXT A (i.e. the AXT showing the largest shift) not only feels a much larger effect from all the residues which are common to both AXTs (such as phenylalanine PHE, and tyrosine, TYR) but it has additional residues contributing significantly (such as an asparagine, ASN and a glutamine, GLN). What mostly differentiate the two chromophores, however, are the electrostatic (and polarization) effects of the residues on the ground state. It appears that AXT B feels a strong effect due to charged (ASP) and polar (SER, THR) residues from both sides of the chain while for AXT A the most interacting residues are placed along the chain (the charged ASP) and on one side (GLN). This asymmetry can explain the much larger bathochromic shift found for AXT A.

This analysis clearly shows that a static representation in terms of a single "frozen" arrangement of the residues leads to significant differences between the two AXTs. To verify if this is physically sound or an artifact, we need to introduce temperature-dependent fluctuations of the system leading to many different AXTenvironment configurations.

To do that, a Born-Oppenheimer (BO) MD simulation has been performed where the two AXTs are described at B3LYP/6-31G level while the protein is represented in terms of the polarizable AMOEBA force field. To perform the QM/AMOEBA BOMD simulation, the recently implemented interface between a locally modified version of Gaussian ${ }^{37}$ and Tinker ${ }^{38}$ has been used (see Section S3 of the SI for more details). ${ }^{39}$

As a preliminary analysis, possible excitonic effects have been checked, calculating the excitation energies of the dimer and the separate monomers on structures randomly extracted from the BO-MD trajectory. In the monomeric calculation, the second AXT is treated on an equal footing with the protein residues. The obtained excitation properties are almost unchanged switching from the dimer to monomers (see also NTO in Section S2 of the SI) and the excitation energies differing for less than $\sim 0.01 \mathrm{eV}$. This confirms the previous studies showing a negligible contribution of excitonic interactions in the observed shift. ${ }^{11,16,21,22}$ Once validated the monomeric model, we have considered 40 uncorrelated configurations extracted from the BO-MD and used them to calculate the electronic excitations of the two AXTs within the polarizable AMOEBA embedding. The results are reported in Figure 3. 


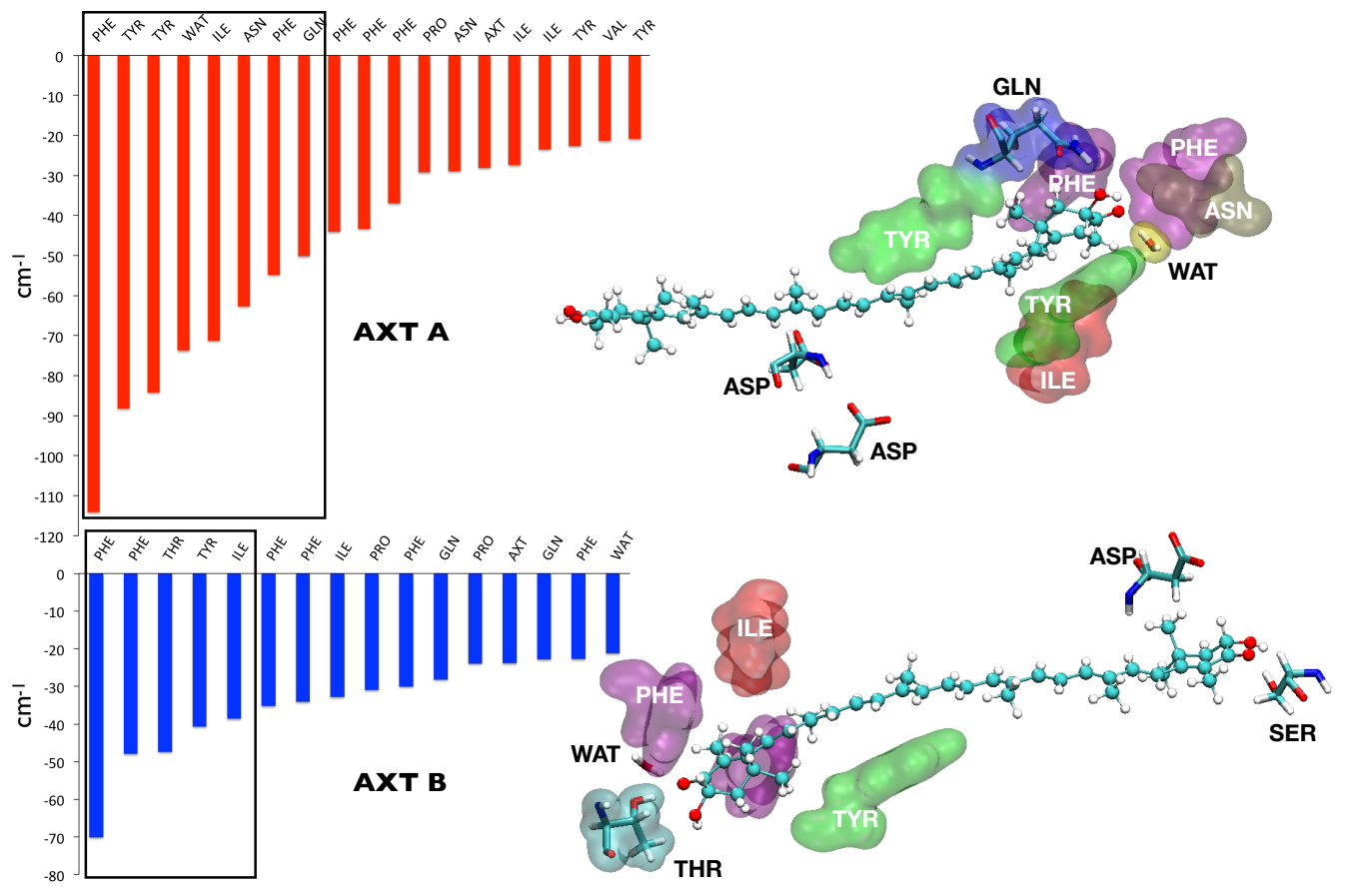

Figure 2: Left: contribution of each residue to the excitation energy of the two AXTs (in $\mathrm{cm}^{-1}$ ). Right: The residues which contribute most are indicated by colored surfaces. A threshold of $40 \mathrm{~cm}^{-1}$ has been used and the depicted residues are those contained in the black box. The residues reported as tubes are those with the largest electrostatic and polarization interaction energy $(>5 \mathrm{Kcal} / \mathrm{mol}$ ) with each AXT in the ground state.

The resulting band maximum has been finally used to calculate the $<$ AMOEBA $>_{M D}$ shift reported in Table 1 . The obtained result $(0.50$ $\mathrm{eV})$ is in excellent agreement with the experimental one of $0.51 \mathrm{eV}$. The remarkable agreement is not limited to the energy shift but it also applies to the absolute value of the excitation energy (2.06 eV vs. $2.10 \mathrm{eV}$ ) and to the band width: the FWHM calculated on the convolution reported in Fig. 3 is $0.51 \mathrm{eV}$ while from the experimental spectrum we get $0.54 \mathrm{eV} .{ }^{15}$

The simultaneous excellent reproduction of these three main features of the protein-induced spectral changes cannot be due to error cancellations but instead confirms the validity of the model thus allowing us to propose a molecular mechanism for the tuning.

First of all, the temperature-dependent fluctuations of the AXT-protein complex act to reduce the large differences found between the two AXTs when simulated within a frozen environment. Now, the chromophores are allowed to explore a common range of local environments as shown by the large overlap of the excitation energies calculated for the two AXTs (see Figure 3). To better understand the role of these structural fluctuations of the chromophores coupled to those of the embedding protein, it is useful to introduce a geometrical parameter largely used in conjugated systems to relate electronic properties to the structure. This parameter is called Bond Length Alternation (BLA) and it is defined as the difference between the average single and the average double bond length along the conjugated chain. The BLA parameter has been successfully used to explain the optical properties of various pigment-protein complexes ${ }^{3,6,40-43}$ but it has never been applied to crustacyanin.

The correlation between the calculated excitation energies and the BLA of the corresponding structures is reported in Figure 3 for the two AXTs. As expected due to the high sensitivity of the excitation energies of carotenoids to changes in the conjugation path, ${ }^{44}$ a linear correlation appears for both AXTs. The slightly different slopes and spread are likely due to the different local environment which we have pre- 

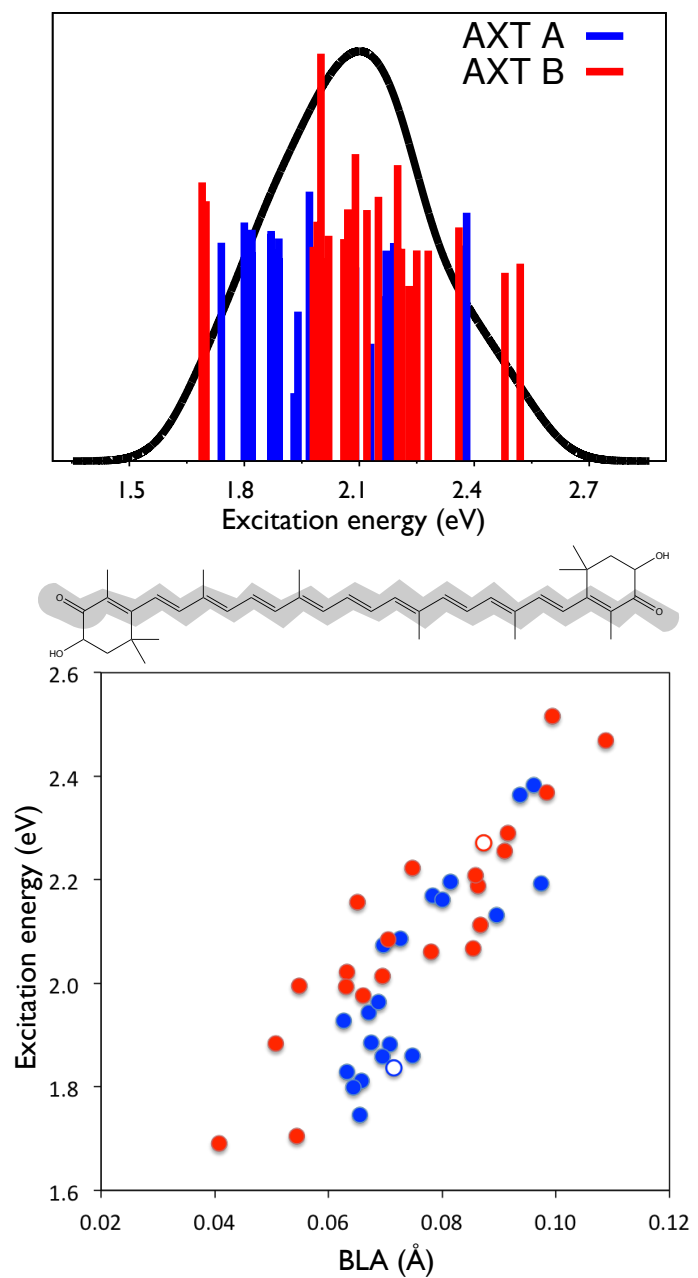

Figure 3: Top: Distribution of the TDDFT/AMOEBA excitation energies on the snapshots extracted along the BOMD: the heights of the sticks are proportional to the squared modulus of the corresponding transition dipoles. The black curve is obtained as the sum of Gaussian convoluted bandshapes centered on each excitation energy and having a FWHM of $500 \mathrm{~cm}^{-1}$ (changing the width from 500 to 1000 $\mathrm{cm}^{-1}$ has a negligible effect on the position of the maximum). Bottom: correlation diagram between the excitation energies (in $\mathrm{eV}$ ) of the two AXTs with their respective BLA (in $\AA$ ) defined using the single and double bonds underlined in gray in the AXT structure. The empty dots refer to the optimized structures within the crystal.

viously discussed in the analysis of the crystal. What is quite unexpected is instead the large window of energies (around $1.0 \mathrm{eV}$ ) that these fluctuations in the BLA allow both AXTs to explore.

To better understand this finding, in Fig. 4 we report the set of residues which mostly acts on AXT A in the crystal structure and in a configuration extracted from the BOMD trajectory which presents characteristics (both in terms of BLA and excitation energy) similar to the average values. The color scheme is the same as the one used in Fig. 2: the residues reported as tubes are those with the largest electrostatic and polarization interaction energy with AXT in the ground state, whereas the residues reported as colored surfaces are those showing the largest response to the excitation. Here, to make the picture more complete, we have used a smaller value for the ground state interaction energy threshold and we have used red(blue) color to indicate residues giving a negative(positive) interaction energy.

As it can be seen, the structure of the chromophore is very different in the two configurations: while in the relaxed crystal structure it is almost straight (with $\mathrm{BLA}=0.051 \AA$ ) in the BOMD configuration it is much more bent (with $\mathrm{BLA}=0.078 \AA$ ). This structural difference is accompanied by a change in the number and the type of interacting residues. In particular, the number of residues having strong interactions (e.g. above the selected threshold) with the ground state is much larger in the crystal structure than in the selected snapshot. We thus expect a much larger stabilization of the ground state in the former. Some differences are also present in the response of the polarizable residues to the excitation: we see that in the crystal structure there are five strongly responsive residues (plus the H-bonded water) while, in the configuration from the BOMD, these reduce to three (and water is no more within the selected threshold). These differences are reflected in the very different energy shift which reduces from $0.83 \mathrm{eV}$ (in the crystal configuration) to $0.46 \mathrm{eV}$ in the BOMD configuration. We can better explain this reduction in terms of different contributions. By calculating the excitation energy of the isolated AXT in the two different geometrical structures we obtain ca. $62 \%$ of the whole reduction. By switching on the effects of the residues we can assign a further $23 \%$ to the different stabilization of the ground state and the remaining $15 \%$ to the 

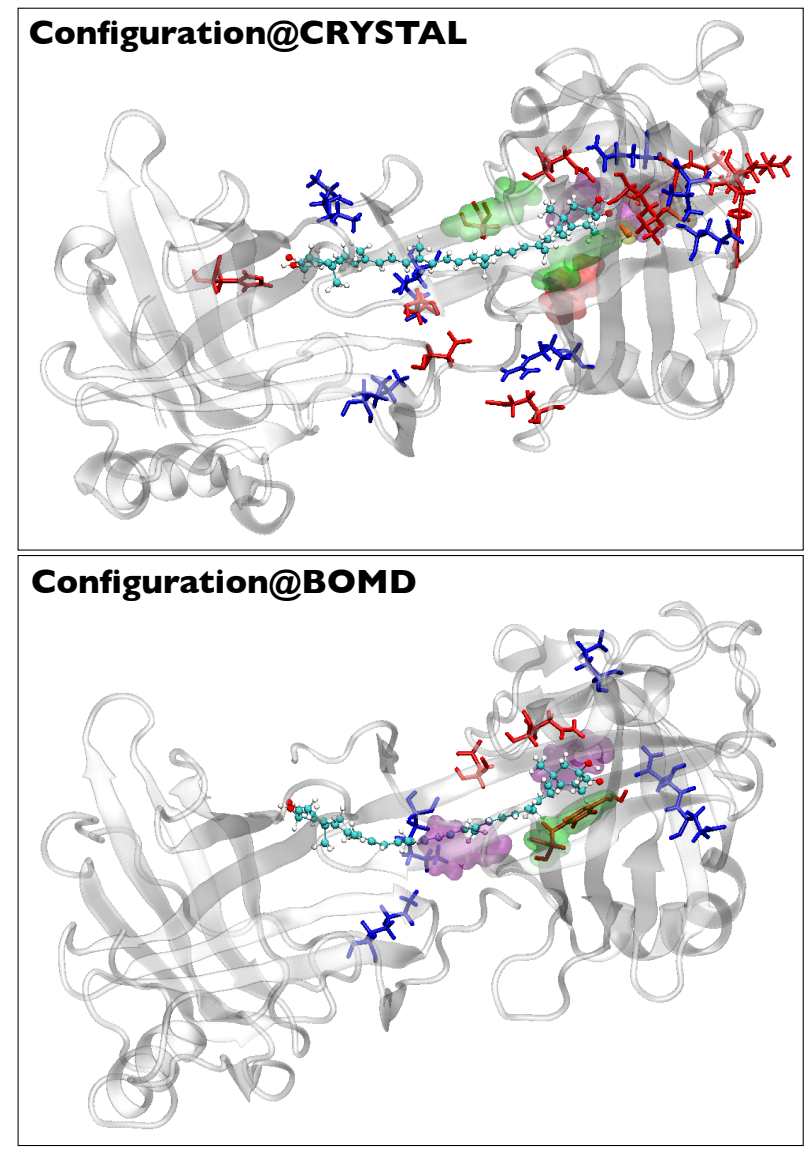

Figure 4: Comparison between $\operatorname{AXT}(\mathrm{A})$ in the crystal (top) and in a configuration extracted from the BOMD (bottom). The residues reported as tubes are those with the largest interaction energy with the AXT in its ground state. Only the residues having an interaction energy of more than $3 \mathrm{kcal} / \mathrm{mol}$ (absolute value) are shown: red means a negative energy while blue means a positive energy. Colored surfaces indicates the residues with the largest response to the excitation. differential response to the excitation.

This analysis clearly says that the protein determines the optical response of the embedded AXTs through fluctuations of the geometrical structure. The resulting conjugation paths will in fact correspond to a different ground state stabilization through the electrostatic (and polarization) effects of the surrounding residues which will differently respond to the excitation process. Without the inclusion of the dynamics, none of these coupled effects can be properly described and only a qualitative picture can be obtained. Moreover, we argue that cumulative electrostatic/polarization effects can also explain the further red shift of $\approx 0.15 \mathrm{eV}$ from $\beta$-crustacyanin to the octamer $\alpha$-crustacyanin.

To further validate the accuracy of the simulations in reproducing the coupling between geometry and environment we have run a short BOMD using a larger basis set (namely 6$311 G$ ): as shown in Section S4 of the SI, the resulting structures reproduce the same range of BLAs and consequently the same distribution of excitation energies. We have also checked the effect of using a polarizable FF by selecting four snapshots from the QM/AMOEBA BOMD characterized by a quite different value of the BLA and, keeping frozen the environment, optimizing the structure of AXT within a nonpolarizable (AMBER) and the AMOEBA protein. As the environment is kept fixed, only the different charges and the presence of polarization will affect the final geometry of the embedded carotenoid. The data (reported in the SI) confirm a non-negligible effect of the polarizable environment: without including polarization, BLA values are always larger which means a larger localization into single and double bonds. These differences in the BLA finally correspond to a 0.1-0.2 eV differences (towards the blue) in the excitation energies.

In conclusion, with the present study we have shown that the color tuning in proteins is a complex phenomenon and its modeling requires to go beyond the methodological approaches commonly used to describe embedded systems. In particular, the popular strategy based on a single relaxed structure (generally obtained from crystal data) cannot be used. Both the 
electronic and the nuclear structure of highly conjugated pigments are in fact so sensitive to the details of the surrounding that a single configuration of the environment necessarily induces artifacts that cannot be removed neither by using a high-level QM description for the pigment and/or introducing an accurate atomistic and polarizable model for the environment. Unfortunately, also the other popular strategy based on the use of a configurational sampling obtained from classical MD simulations presents serious limitations. In this case, in fact, an accurate enough description of the coupled torsional and bond-length distorsions of the pigments' conjugated structure is difficult to be achieved. ${ }^{45} \mathrm{~A}$ complete and reliable picture can be obtained only if the pigment's electronic and structural fluctuations induced by the dynamic electrostatic fields of the protein and the residue-dependent polarization interactions can be accurately included in all the steps of the simulation. This necessarily requires to apply dynamic QM/classical approaches where the classical models include all the main features which define the embedding effects of a protein, namely an atomistic detail combined with an accurate description of electrostatics and polarization.

\section{Supporting Information Avail- able}

Details on the approach employed to include the effect of the polarizable environment on the electronic excitation. Test calculations for the $\omega$ B97X DFT functional and details about the QM/MM BOMD. $\omega$ B97X DFT NTOs of AXT. Test calculations on the effect of the basis set and the polarizable FF

Acknowledgement FB acknowledges the use of supercomputer facilities at SURFsara sponsored by NWO Physical Sciences, with financial support from the Netherlands Organization for Scientific Research (NWO).

\section{References}

(1) Tadepalli, S.; Slocik, J. M.; Gupta, M. K.; Naik, R. R.; Singamaneni, S. BioOptics and Bio-Inspired Optical Materials. Chem. Rev. 2017, 117, 12705-12763.

(2) Hoffmann, M.; Wanko, M.; Strodel, P.; König, P. H.; Frauenheim, T.; Schulten, K.; Thiel, W.; Tajkhorshid, E.; Elstner, M. Color Tuning in Rhodopsins: The Mechanism for the Spectral Shift between Bacteriorhodopsin and Sensory Rhodopsin II. J. Am. Chem. Soc. 2006, 128, 10808-10818.

(3) Altun, A.; Yokoyama, S.; Morokuma, K. Color Tuning in Short WavelengthSensitive Human and Mouse Visual Pigments: Ab initio Quantum Mechanics/Molecular Mechanics Studies. J. Phys. Chem. A 2009, 113, 11685-11692.

(4) Philip, A. F.; Nome, R. A.; Papadantonakis, G. A.; Scherer, N. F.; Hoff, W. D. Spectral tuning in photoactive yellow protein by modulation of the shape of the excited state energy surface. Proc. Natl. Acad. Sci. USA 2010, 107, 5821-5826.

(5) Hasegawa, J.; Fujimoto, K. J.; Nakatsuji, H. Color Tuning in Photofunctional Proteins. ChemPhysChem 2011, 12, 3106-3115.

(6) Murugan, N. A.; Kongsted, J.; Rinkevicius, Z.; Agren, H. Color modeling of protein optical probes. Phys. Chem. Chem. Phys. 2012, 14, 1107-1112.

(7) Mendes-Pinto, M. M.; LaFountain, A. M.; Stoddard, M. C.; Prum, R. O.; Frank, H. A.; Robert, B. Variation in carotenoid-protein interaction in bird feathers produces novel plumage coloration. J. R. S. Interface 2012, 9, 3338-3350.

(8) Cheng, C.; Kamiya, M.; Uchida, Y.; Hayashi, S. Molecular Mechanism of Wide Photoabsorption Spectral Shifts of Color 
Variants of Human Cellular Retinol Binding Protein II. J. Am. Chem. Soc. 2015, 137, 13362-13370.

(9) Udvarhelyi, A.; Olivucci, M.; Domratcheva, T. Role of the Molecular Environment in Flavoprotein Color and Redox Tuning: QM Cluster versus QM/MM Modeling. J. Chem. Theory Comput. 2015, 11, 3878-3894.

(10) Begum, S.; Cianci, M.; Durbeej, B.; Falklof, O.; Hadener, A.; Helliwell, J. R.; Helliwell, M.; Regan, A. C.; Watt, C. I. F. On the origin and variation of colors in lobster carapace. Phys. Chem. Chem. Phys. 2015, 17, 1-10.

(11) Gamiz-Hernandez, A. P.; Angelova, I. N.; Send, R.; Sundholm, D.; Kaila, V. R. I. Protein-Induced Color Shift of Carotenoids in $\beta$-Crustacyanin. Angew. Chem. 2015, 127, 11726-11729.

(12) Katayama, K.; Okitsu, T.; Imai, H.; Wada, A.; Kandori, H. Identical Hydrogen-Bonding Strength of the Retinal Schiff Base between Primate Green- and Red-Sensitive Pigments: New Insight into Color Tuning Mechanism. $J$. Phys. Chem. Lett. 2015, 6, 1130-1133.

(13) Hense, A.; Nienhaus, K.; Nienhaus, G. U. Exploring color tuning strategies in red fluorescent proteins. Photochem. Photobiol. Sci. 2015, 14, 200-212.

(14) Deisseroth, K.; Hegemann, P. The form and function of channelrhodopsin. Science 2017, 357, eann5544.

(15) van Wijk, A. A. C.; Spaans, A.; Uzunbajakava, N.; Otto, C.; de Groot, H. J. M.; Lugtenburg, J.; Buda, F. Spectroscopy and Quantum Chemical Modeling Reveal a Predominant Contribution of Excitonic Interactions to the Bathochromic Shift in $\alpha$-Crustacyanin, the Blue Carotenoprotein in the Carapace of the Lobster Homarus gammarus. J. Am. Chem. Soc. 2005, 127, 1438-1445.
(16) Neugebauer, J.; Veldstra, J.; Buda, F. Theoretical Spectroscopy of Astaxanthin in Crustacyanin Proteins: Absorption, Circular Dichroism, and Nuclear Magnetic Resonance. J. Phys. Chem. B 2011, 115, 3216-3225.

(17) Cianci, M.; Rizkallah, P. J.; Olczak, A.; Raftery, J.; Chayen, N. E.; Zagalsky, P. F.; Helliwell, J. R. The molecular basis of the coloration mechanism in lobster shell: beta-crustacyanin at 3.2-A resolution. Proc. Natl. Acad. Sci. USA 2002, 99, 9795-9800.

(18) Durbeej, B.; Eriksson, L. A. On the bathochromic shift of the absorption by astaxanthin in crustacyanin: a quantum chemical study. Chem. Phys. Lett. 2003, 375, 30-38.

(19) Bartalucci, G.; Coppin, J.; Fisher, S.; Hall, G.; Helliwell, J. R.; Helliwell, M.; Liaaen-Jensen, S. Unravelling the chemical basis of the bathochromic shift in the lobster carapace; new crystal structures of unbound astaxanthin, canthaxanthin and zeaxanthin. Acta Crystallogr. B 2007, 63, 328-337.

(20) Ilagan, R. P.; Christensen, R. L.; Chapp, T. W.; Gibson, G. N.; Pascher, T.; Poívka, T.; Frank, H. A. Femtosecond Time-Resolved Absorption Spectroscopy of Astaxanthin in Solution and in $\alpha-$ Crustacyanin. J. Phys. Chem. A 2005, 109, 3120-3127.

(21) Strambi, A.; Durbeej, B. Excited-State Modeling of the Astaxanthin Dimer Predicts a Minor Contribution from Exciton Coupling to the Bathochromic Shift in Crustacyanin. J. Phys. Chem. B 2009, 113, 5311-5317.

(22) Christensson, N.; Žídek, K.; Magdaong, N. C. M.; LaFountain, A. M.; Frank, H. A.; Zigmantas, D. Origin of the Bathochromic Shift of Astaxanthin in Lobster Protein: 2D Electronic Spectroscopy Investigation 
of $\beta$-Crustacyanin. J. Phys. Chem. B 2013, 117, 11209-11219.

(23) Britton, G.; Weesie, R. J.; Askin, D.; Warburton, J. D.; Gallardo-Guerrero, L.; Jansen, F. J.; de Groot, H. J. M.; Lugtenburg, J.; Cornard, J.-P.; Merlin, J.C. Carotenoid Blues: Structural Studies on Carotenoproteins. Pure Appl. Chem. 1997, 69, 2075-2084.

(24) Götze, J. P.; Thiel, W. TD-DFT and DFT/MRCI study of electronic excitations in Violaxanthin and Zeaxanthin. Chem. Phys. 2013, 415, 247-255.

(25) Ostroumov, E.; Müller, M. G.; Marian, C. M.; Kleinschmidt, M.; Holzwarth, A. R. Electronic Coherence Provides a Direct Proof for Energy-Level Crossing in Photoexcited Lutein and $\beta$-Carotene. Phys. Rev. Lett. 2009, 103, 108302.

(26) Spezia, R.; Knecht, S.; Mennucci, B. Excited state characterization of carbonyl containing carotenoids: a comparison between single and multireference descriptions. Phys. Chem. Chem. Phys. 2017, 19, 17156-17166.

(27) Götze, J. P.; Kröner, D.; Banerjee, S.; Karasulu, B.; Thiel, W. Carotenoids as a Shortcut for Chlorophyll Soret-to-Q Band Energy Flow. Chem. Phys. Chem. 2014, 15, 3392-3401.

(28) Spezia, R.; Knecht, S.; Mennucci, B. Excited state characterization of carbonyl containing carotenoids: a comparison between single and multireference descriptions. Phys Chem Chem Phys 2017, 19, 17156-17166.

(29) Chai, J.-D.; Head-Gordon, M. Systematic optimization of long-range corrected hybrid density functionals. J. Chem. Phys. 2008, 128, 084106.

(30) Zagalsky, P. F. $\beta$-Crustacyanin, the bluepurple carotenoprotein of lobster carapace: consideration of the bathochromic shift of the protein-bound astaxanthin. Acta Crystallogr. D 2003, 59, 1529-1531.

(31) Polívka, T.; Frank, H. A.; Enriquez, M. M.; Niedzwiedzki, D. M.; Liaaen-Jensen, S.; Hemming, J.; Helliwell, J. R.; Helliwell, M. X-ray Crystal Structure and Time-Resolved Spectroscopy of the Blue Carotenoid Violerythrin. J. Phys. Chem. B 2010, 114, 8760-8769.

(32) Tomasi, J.; Mennucci, B.; Cammi, R. Quantum Mechanical Continuum Solvation Models. Chem. Rev. 2005, 105, 2999 3094 .

(33) Caricato, M.; Mennucci, B.; Tomasi, J.; Ingrosso, F.; Cammi, R.; Corni, S.; Scalmani, G. Formation and relaxation of excited states in solution: A new time dependent polarizable continuum model based on time dependent density functional theory. J. Chem. Phys. 2006, 124, 124520-13.

(34) Ponder, J. W.; Wu, C.; Ren, P.; Pande, V. S.; Chodera, J. D.; Schnieders, M. J.; Haque, I.; Mobley, D. L.; Lambrecht, D. S.; DiStasio Jr, R. A. et al. Current Status of the AMOEBA Polarizable Force Field. $J$. Phys. Chem. B 2010, 114, 2549-2564.

(35) Loco, D.; Polack, É.; Caprasecca, S.; Lagardère, L.; Lipparini, F.; Piquemal, J.P.; Mennucci, B. A QM/MM Approach Using the AMOEBA Polarizable Embedding: From Ground State Energies to Electronic Excitations. J. Chem. Theory Comput. 2016, 12, 3654-3661.

(36) Guareschi, R.; Valsson, O.; Curutchet, C.; Mennucci, B.; Filippi, C. Electrostatic versus Resonance Interactions in Photoreceptor Proteins: The Case of Rhodopsin. J. Phys. Chem. Lett. 2016, 7, 4547-4553.

(37) Frisch, M. J.; Trucks, G. W.; Schlegel, H. B.; Scuseria, G. E.; 
Robb, M. A.; Cheeseman, J. R.; Scalmani, G.; Barone, V.; Mennucci, B.; Petersson, G. A. et al. Gaussian Development Version, Revision H.36. Gaussian Inc. Wallingford CT 2010.

(38) Ponder, J. W. TINKER, Software Tools for Molecular Design. http://dasher. wustl.edu/tinker.

(39) Loco, D.; Lagardère, L.; Caprasecca, S.; Lipparini, F.; Mennucci, B.; Piquemal, J.P. Hybrid QM/MM Molecular Dynamics with AMOEBA Polarizable Embedding. J. Chem. Theory Comput. 2017, 13, 4025-4033.

(40) Murugan, N. A.; Kongsted, J.; Rinkevicius, Z.; Ågren, H. Breakdown of the first hyperpolarizability/bond-length alternation parameter relationship. Proc. Natl. Acad. Sci. USA 2010, 107, 1645316458.

(41) Drobizhev, M.; Hughes, T. E.; Stepanenko, Y.; Wnuk, P.; O'Donnell, K.; Scott, J. N.; Callis, P. R.; Mikhaylov, A.; Dokken, L.; Rebane, A. Primary Role of the Chromophore Bond Length Alternation in Reversible Photoconversion of Red Fluorescence Proteins. Sci. Rep. 2012, 2, 969-6.

(42) Daday, C.; Curutchet, C.; Sinicropi, A.; Mennucci, B.; Filippi, C. ChromophoreProtein Coupling beyond Nonpolarizable Models: Understanding Absorption in Green Fluorescent Protein. J. Chem. Theory Comput. 2015, 11, 4825-4839.

(43) Buda, F.; Keijer, T.; Ganapathy, S.; de Grip, W. J. A Quantum-mechanical Study of the Binding Pocket of Proteorhodopsin: Absorption and Vibrational Spectra Modulated by Analogue Chromophores. Photochem Photobiol 2017, 93, 1399-1406.

(44) Llansola-Portolés, M. J.; Pascal, A. A.; Robert, B. Electronic and vibrational properties of carotenoids: from in vitro to in vivo. J. R. Soc. Interface 2017, 14, 20170504.

(45) Andreussi, O.; Prandi, I. G.; Campetella, M.; Prampolini, G.; Mennucci, B. Classical Force Fields Tailored for QM Applications: Is It Really a Feasible Strategy? J. Chem. Theory Comput. 2017, 13, 4636-4648. 
Graphical TOC Entry

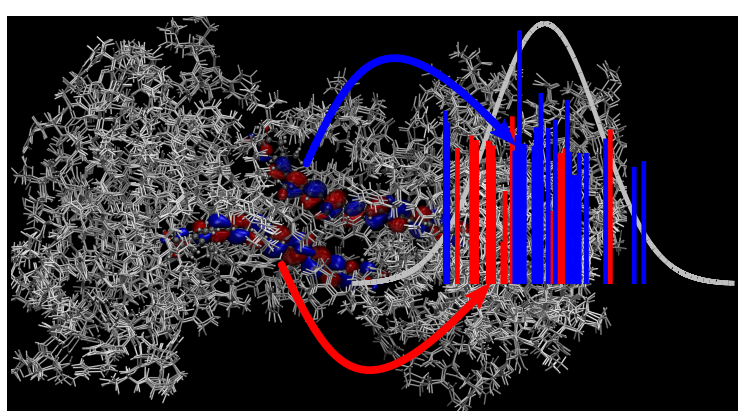

